\title{
Evaluation of material's state during deformation process by means of thermographic measurement
}

\author{
by M. Žd'árský, J. Valach**, D. Kytýřr \\ * Faculty of Transportation Sciences, Czech Technical University, Konviktská 20, 11000 Prague 1, Czech \\ Republic, \{xzdarsky,kytyr\}@fd.cvut.cz \\ ** Institute of Theoretical and Applied Mechanics, AS CR, v.v.i., Prosecká 76, 19000 Prague 9, Czech \\ Republic, valach@itam.cas.cz
}

\begin{abstract}
In this paper the application of quantitative thermography analysis on strains is presented on the study dealing with evaluation of material's behavior during plastic deformation of specimen in two thermodynamically different states. The analysis of plastic deformation and thermal phenomena processes is demonstrated on compact-tension steel specimens - both in theoretical and experimental way. The experiments proved different temperature response to external loading and dependence of plastic deformation development on material's state. These results support claim that thermography can be used for non-destructive investigation of internal state of materials.
\end{abstract}

\section{Introduction}

Behavior of materials sustaining large strains is of great importance not only in shaping processes during manufacturing but also in study of materials' ability to retain their ductility in advanced stages of deformation. Therefore detailed investigation of deformation's evolution is an important experimental task which can be carried out by various techniques and non-destructive or even non-invasive methods play a prominent role among them.

If the object's shape is complex or its material is structured or inhomogeneous or the deformation itself develops unevenly, the strains cannot be represented by one value for the whole object and have to be recorded as a field of values in order to pinpoint local variations. To achieve this goal, techniques capable of detection of the whole array of measured values are the only choice. Optical methods, like digital image correlation, are frequently exploited in experimental mechanics, but in this case large deformations in object prohibit their application. This obstacle can be easily overcome by thermography that can also provide insight into the processes which the studied material undergoes [1]. Thermography is a suitable method for experimental investigation whenever there is an energy conversion in the observed process accompanied with transfer, release or absorption of heat. This condition is fulfilled in loading process as a coupling between mechanical stress and temperature change, thermoelasticity [2, 3], takes part in the first stage of loading which happens elastically, it means reversibly. In this stage of loading temperature decreases as tensile stresses in the specimen cause volume expansion associated with adiabatic cooling. The temperature decrease is stopped and then reversed in the process of continuing loading when the yield stress in material is achieved. At this stage the specimen's material deforms plastically. The process of plastic deformation is in metals at crystallographic level carried out by movement of dislocations associated with lattice vibration and local increase of temperature [4]. Balance between the two processes - thermoelasticity and thermoplasticity can therefore elucidate internal state of the studied material, especially yield strength parameter.

\section{Analysis of thermoplastic phenomenon on compact-tension geometry specimen}

This section analyses a thermomechanical phenomenon which occurs during its tensile loading in location of stress concentrator which is in the presented case in front of the notch in the axis of a compact tension (CT) specimen. The whole process is controlled by three variables - time, temperature and distance between the boundary of plastic zone and the tip of the notch. There are two reasons why limit investigation to set of the points on symmetry axis of the specimen - the obvious one is the symmetry itself and the second one is that also stresses, strains and temperatures reach their maximal values there. In order to compare contribution of thermoelastic and thermoplastic effect to the temperature changes, loading process has to be analyzed. It follows from cross-head speed $v=2 \mathrm{~mm} / \mathrm{min}$ and geometry of the specimen ( $/$ is distance between loading openings in the specimen), the strain rate is roughly $\dot{\varepsilon} \cong v / l$, in our case equals to $1 \%$ /s. Elastic strain at the yield point is $\varepsilon_{e l_{-} \max }=\sigma_{k} / E$ which is upper bound of the estimate and in reality is less than this value. This strain is attained at the time $t=\varepsilon_{e l_{-} \max } / \dot{\varepsilon}$, approximately in 2s, but the loading is carried out for several minutes, therefore the elastic strain is only a minute fraction of the total strain. Therefore ideal elasto-plastic material model implies that thermoelastic decrease of temperature is almost immediately precluded by heat release due to yield. Stress on the axis remains constant without hardening, it means it retains elastic state till reaching yield point. 
The description and the analysis of a plastic behavior starts at time $t=t_{0}$, when the yield strength is achieved and specimen starts to deform plastically at the point $x=0=x_{0}$. At this time, temperature is equal to the ambient temperature $T_{0}$ in the entire specimen. Origin of coordinate system of time - distance - temperature space describing studied phenomena is therefore set to $\left[t_{0}, T_{0}, x_{0}\right]$. The applied force of a testing machine produces the concentration of stress at the notch and then plastic deformation further propagates. The extent of plastic deformation is expressed by the distance between the boundary of a plastic zone and the notch - drawn as a pink curve. The border of the plastic zone reaches distance $x_{1}$ at time $t_{1}$. This plastic region is related to the increase of deformation $\Delta \varepsilon_{1-0}$ in time interval from $t_{1}$ to $t_{0}$. As a result of the thermomechanical coupling, the deformation caused by the loading for a duration $\Delta t$ (minimum of $\Delta t$ is given by image acquisition frequency of thermal camera) is proportional to a temperature response $\Delta T=T_{1}$. It means that the ratio of temperature increase to plastic deformation increase is constant for materials without hardening. This relation can be expressed as $\Delta T=\left(\sigma_{K} \Delta \varepsilon_{p l}\right) /\left(\rho c_{p}\right)$, where $\sigma_{K}$ is a symbol for yield stress, $\Delta \varepsilon_{p l}$ is a change of plastic deformation, $\rho$ is material's density and $c_{p}$ is specific heat capacity at constant pressure. The temperature as a function of time and location is shown on figure 1 . by the red line starting from the origin. The curve connects the origin with a point $\left[x_{0}, t_{1}, T_{1}\right]$. Temperature distribution on the axis is depicted in figure 1 at time $t=t_{1}$ as vertically hatched area. Similarly, other curves can be drawn describing time evolution of temperature at points $x_{1}$ and $x_{2}$, etc. Correction to thermoelastic effect mentioned above would shift the whole time-location surface of temperature down by fraction of degree of Celsius and therefore is omitted.

In addition to heat sources mentioned at the end of the previous paragraph, the figure 1 does not describe the situation completely, because it does not consider the influence of a heat transfer by conduction. This correction is then shown in figure 2. The temperature increase due to plastic deformation is accompanied with a heat flow to the colder region. It means that the temperature decreases to the value $T_{2-1}$ at the $x=x_{0}$ at time $t_{2}$ while in the rest of examined profile the temperature rises, the extent of temperature decrease or increase is controlled by heat balance and thermo-mechanical properties of the material. This phenomenon is illustrated by set of decreasing green curves in figure 2. This heat transfer is proved by increase in temperature outside the plastic zone. The most distant point, where temperature rise is detectable by thermal camera, is $x_{3}$ at time $t_{2}$. The total temperature change has two major components: heat release associated with plastic zone $\Delta \varepsilon_{1-0}$ (its size was $x_{1}$ at time $t_{1}$ ) and a contribution which comes from thermal conductivity (to the point $x_{3}$ ). This summary is graphically presented in figure 2 .

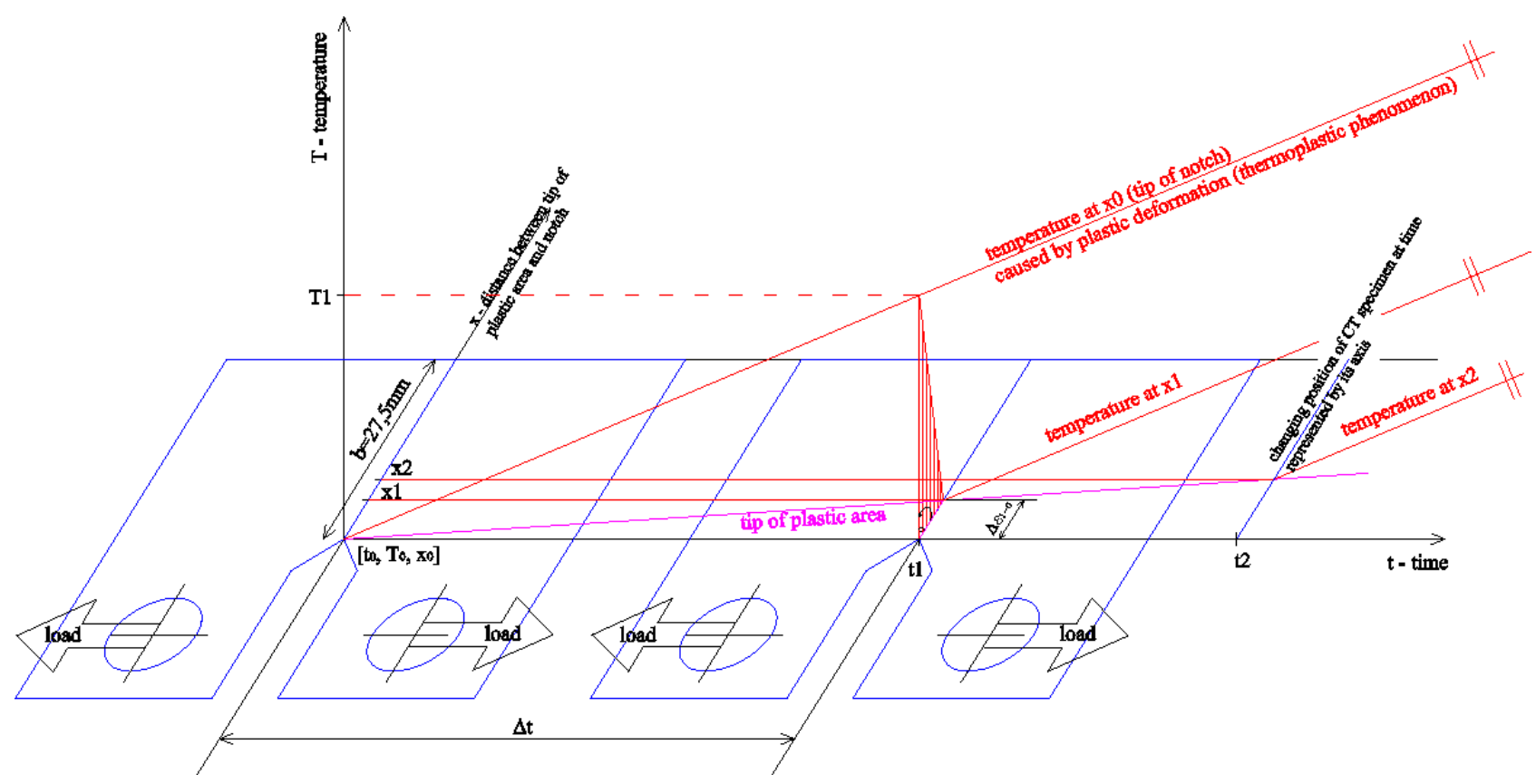

Fig. 1. Development of the temperature in the axis of the CT specimen caused by the thermoplastic phenomenon (without hardening of material, elastic deformation and conduction of heat). For the elucidation of temperature field temporal development, the figure shows the same specimen at two different time moments and its respective temperature field. 


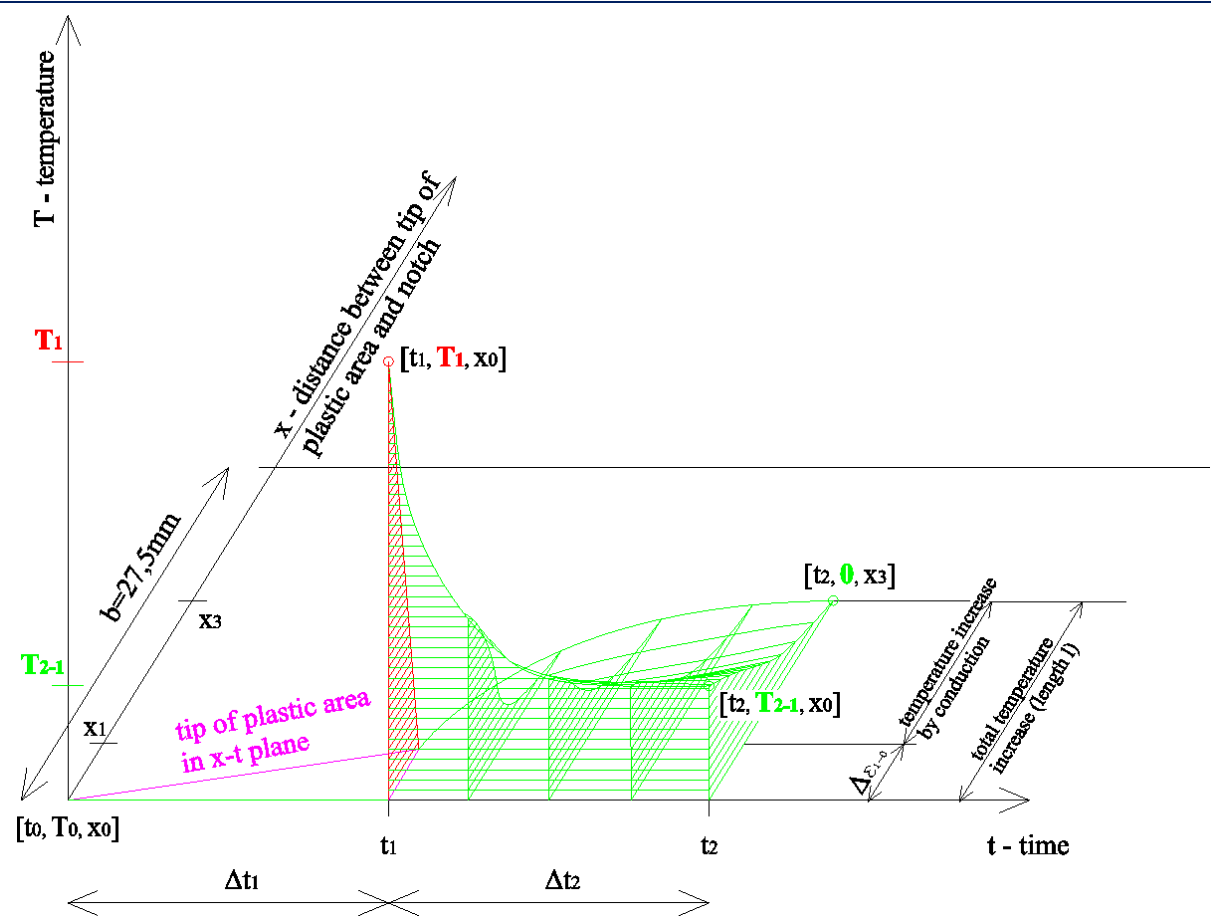

Fig. 2. Contribution of heat conduction to temporal evolution of temperature field in the axis of the CT specimen after interruption of loading.

\section{Experimental}

Studied objects, standard CT specimens were made of the same plain steel, but treated differently. One specimen was left in "as produced" state, while the other was annealed at $700^{\circ} \mathrm{C}$ for four hours and then gradually cooled back to room temperature. As expected, annealing softened the material, i.e. its yield strength decreased. Microscopically, mechanical properties were changed as a result of dislocation density reduction. Lower density of lattice defects effectively decreases the yield stress at which material deforms plastically. Plastic reserve, the total amount of plastic strain the material is able to withstand, has increased at the same time. The two specimens were then loaded in testing machine at the same predefined velocity of crosshead. The loading was interrupted at predefined displacement, prior breaking the specimen in two parts. The intention of this experiment was twofold: to verify effect of different thermal treatment of material on its response to external load and also reverse task to estimate capability of the thermography method to detect differences in behavior of the specimens and therefore to distinguish between the two different states of the material.

\subsection{Mechanical loading}

The two differently thermally treated specimens were subjected to the same loading test. In the course of the experiment displacement and applied force were recorded simultaneously as well as the sequence of thermographic images was captured.

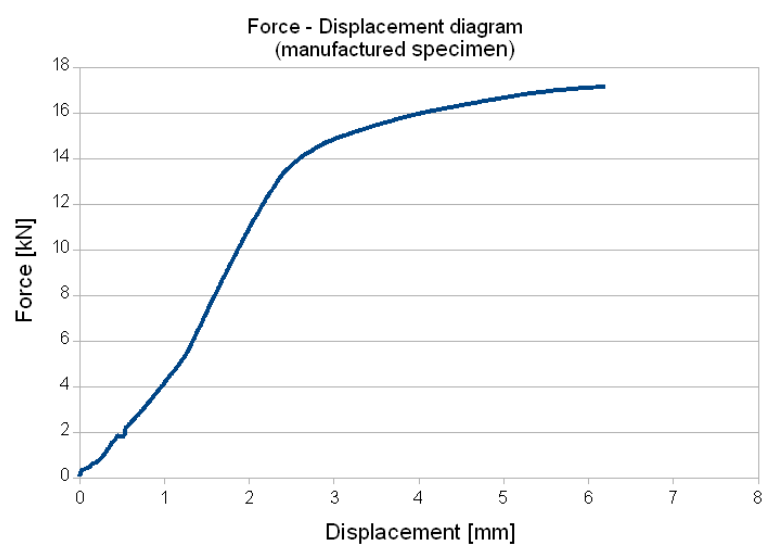

Fig. 3. Force-displacement curve for "as manufactured" (non-treated) material

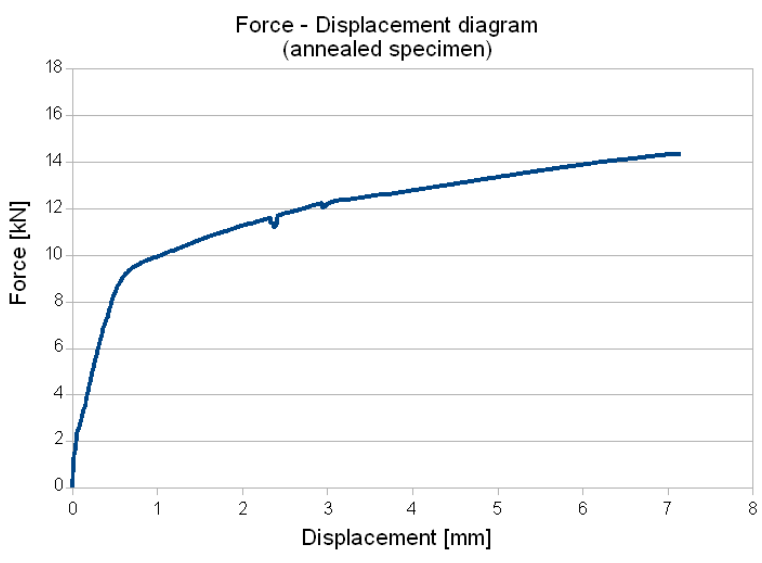

Fig. 4. Force-displacement curve for annealed material 
The two force-displacement curves are clearly distinguishable one from other as can be seen by comparison of figures 3 and 4. The yield strength of "as manufactured" material $R_{m}=660 \mathrm{MPa}(H V 30=210)$ and the yield strength of the annealed material to $\mathrm{R}_{\mathrm{m}}=377 \mathrm{MPa}(\mathrm{HV} 30=114)$ were determined on the basis of Vicker's hardness test. Distinct character of the two materials is noticeable not only in the loading diagrams, but also in thermograms as discussed in the next paragraph.

\subsection{Investigation of plastic zone by means of temperature field analysis}

From analysis of thermograms it follows that the annealed material reacted on absorbed deformation energy by noticeable extension of the plastic zone (in comparison to non-treated specimen) in front of the notch (see figure 5. - on the left - a large plastic zone covers large part of the specimen axis, but is confined in narrow strip along the axis and a minor temperature gradient can be observed). For the second specimen, behavior is quite different. Extension of crack from the notch is detected just after the beginning of the test, indicating low ductility of "as manufactured" state of the material. In the thermogram, this behavior translates into a localized plastic zone and significant temperature gradient (see figure 5 - on the right).
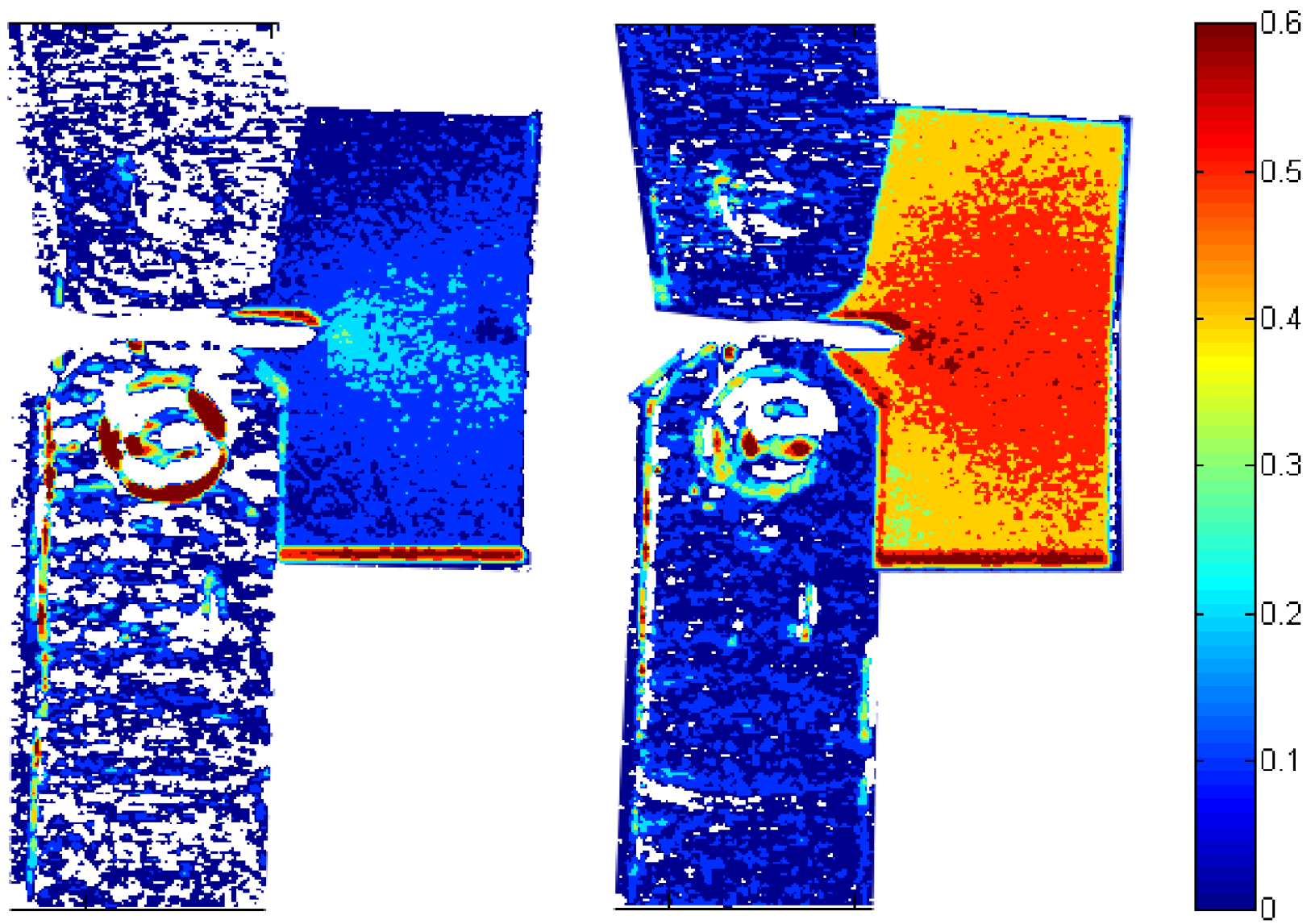

Fig. 5. Comparison of the temperature increase in the CT specimens of the same material but differently treated. Softer, annealed material is on the left, material in "as manufactured" state is on the right. It can be seen that in annealed specimen plastic hinge develops only in narrow strip in the axis of specimen, while the plastic deformation in harder state extends over large area.

Dissipated energy is related to the yield stress magnitude, therefore in the softer material less thermal energy is released, resulting in more gradual and even increase in temperature. While the temperature increases significantly in front of the notch in the "as manufactured" specimen due to higher yield strength, the softer annealed specimen preferred plastic deformation in larger part of the specimen's axis and therefore the temperature gradients were not so pronounced.

There is also a difference in time-span of recorded test. While in the "as manufactured" specimen plastic zone growth happens suddenly (as seen on figure 5), in the case of annealed material the zone is localized and temperature gradient is not so steep, because the heat conduction maintains balance of temperature. The velocity of cross-head (figure 6) movement is noticeable on the opening in front of the notch during the time depicted on the figure. 


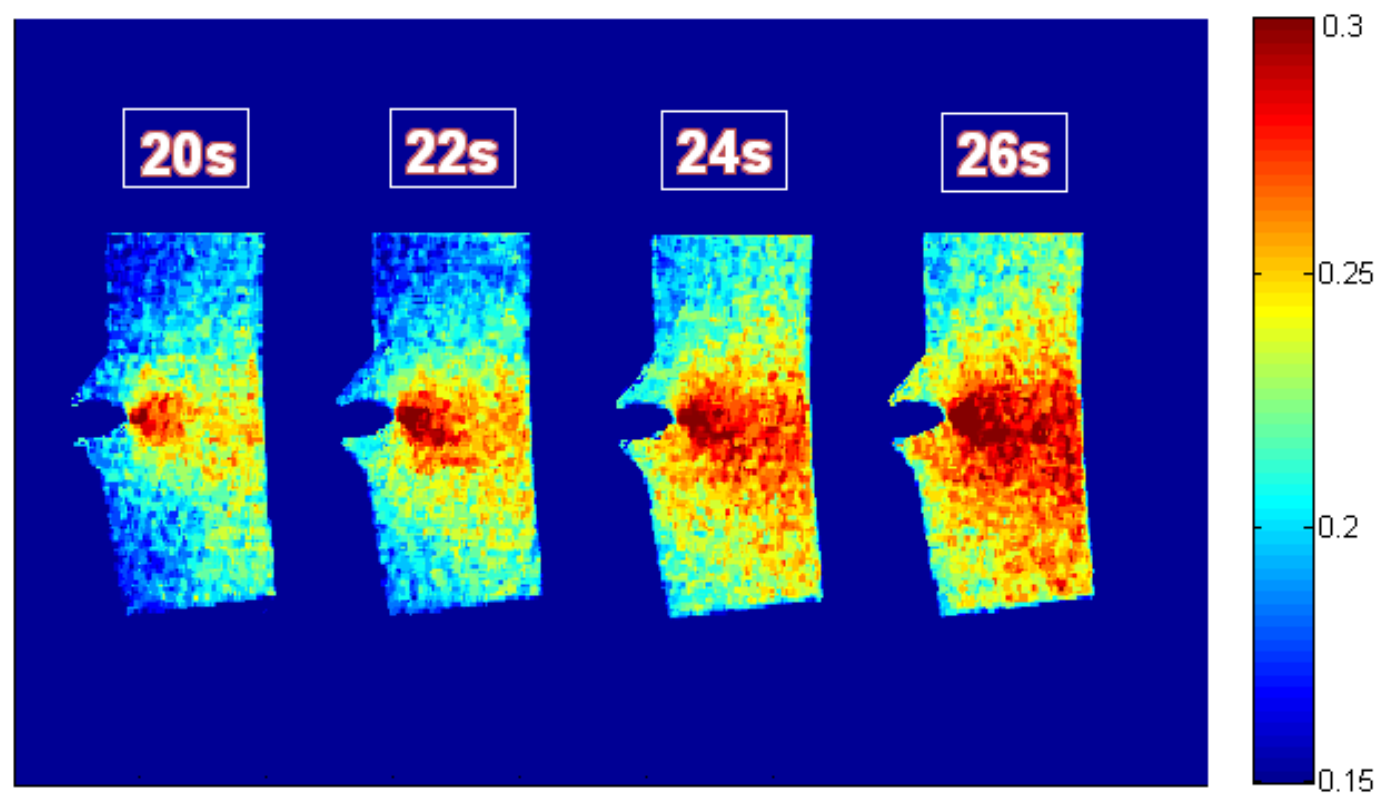

Fig. 6 Temperature evolution during loading of specimen in "as manufactured" state - thermograms sequence starts at time $t=20$ s and time step is $2 \mathrm{~s}$.

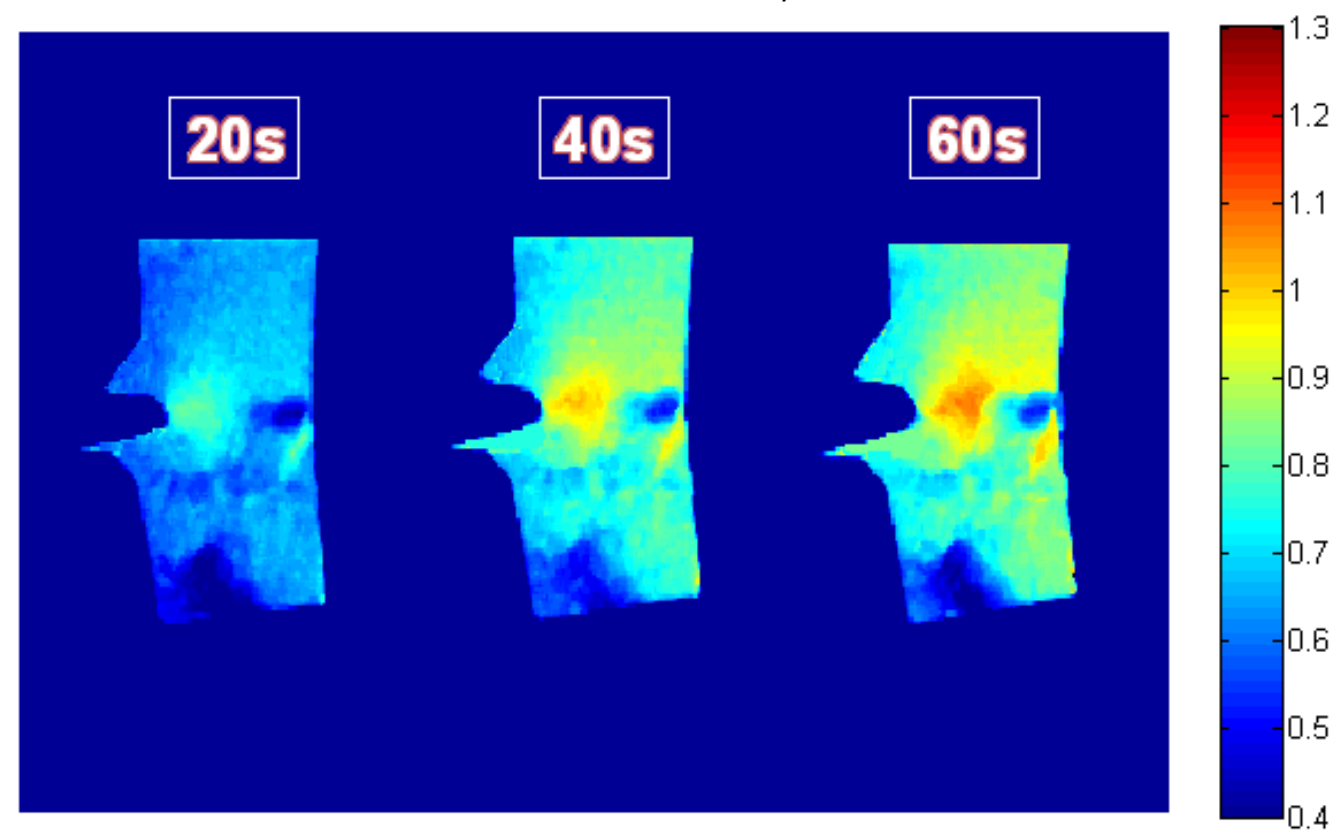

Fig. 7 Temperature evolution during loading of specimen in annealed state - thermograms sequence starts at time $t=20$ s and time step is 20 s. Notice different temperature scale from previous figure.

\subsection{Complementary estimation of plastic zone estimated from simplified plastic joint analysis}

Statements derived from analysis of thermograms sequence can also be supported by independent evidence, based on the study of plastic behavior of specimens. The whole stress and strain field can be calculated more precisely using finite element method [5], but for the purpose of demonstrating strong relation between observed temoerature field and stress distribution, it is sufficient to rely on rough elasto-plastic description of bending.

We assume that a) sum of stresses across the CT specimen area is equal to loading force in the axis of specimen, b) that moment of force implied by distance between applied force and tip of notch has to be in equilibrium with moments of stresses across cross-section and c) that the material is elastic plastic without hardening, it means it retains elastic state till reaching yield point, then the stress remain constant. 
Results of the analysis are plotted in figure 8. On the background outline of CT specimen is drawn and two stress profiles are shown. Comparison of plastic hinge developed in the course of loading for "as manufactured" (= blue line) and annealed specimen ( $=$ red line) reveals influence of material state on its mechanical behavior. It is immediately clear that for annealed specimen the axis if fully plasticized, while the second specimen exhibits only partially plasticized cross section. This calculation is in full agreement with thermography observation.

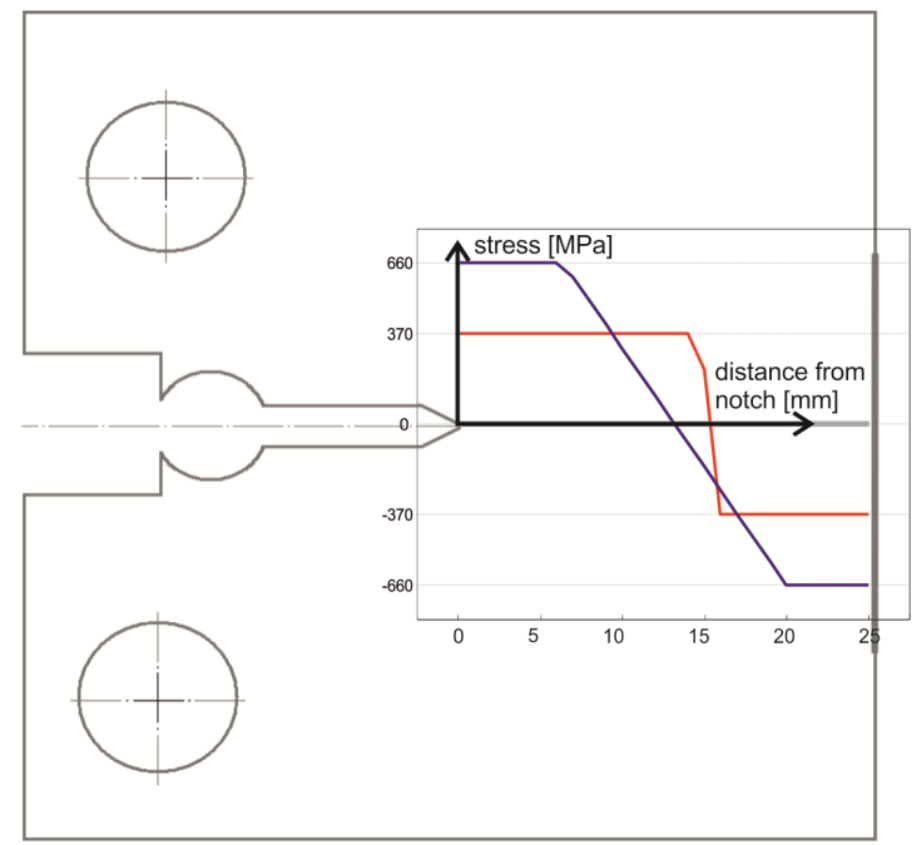

Fig. 8. Stress field along axis confirms development of plastic hinge. Annealed specimen (red line) is nearly plasticized, in the second specimen (blue line), plastic zones are localized only on the edges of the specimens cross section

\section{Conclusions}

Plastic properties are usually determined by specialized mechanical tests, but in some cases thermal camera offers non-conventional means. Thermal camera application allows to distinguish portion of elastic and plastic deformation during loading and to determine work hardening of the tested material. The above described experiment proved assumption that it is possible to use a thermal camera for investigation of material's state. Using thermography it was possible to demonstrate that material in the softer (annealed) state deformed in such a way that only a narrow band of plastic deformation and plastic joints developed, while for the material in "as manufactured" state the zone of plastic deformation is considerably larger, as seen on the figure 1.

Thermography has proved to be a very useful tool in analysis of thermal field changes associated with acting forces causing deformation of studied specimen. Thermal camera is thus indispensable tool in investigation of any process where energy transformation takes part and part of the energy is lost in the form of heat.

Ability of the technique to distinguish material state is important for use of thermography as non-destructive inspection tool capable to detect weakened spots in structure subjected to varying loading. These spots can be results of imperfect manufacturing or material degradation processes like corrosion. The above described experimental approach can be for example used in pipelines as an inspection tools.

\section{Acknowledgement}

This work has been supported by the Grant Agency of the CR (grant No.103/09/2101), Grant Agency of the CTU (grant No. SGS11/205/OHK2/3T/16) and by RVO: 68378297.

\section{REFERENCES}

[1] Vollmer M., Mőllmann K.-P., "Infrared Thermal Imaging". Wiley-VCH, Weinheim, 2010, ISBN: 978-3-527-40717-0.

[2] Tomlinson R. A., Olden E.J., "Thermoelasticity for the analysis of crack tip stress fields". Strain, 35, 2, pp. 49-55, 1999.

[3] Sun X. W. et al., "Quantitative plastic deformation analysis by means of thermoplastic effects". Elsevier Science Publishers, pp. 213-218, Beijing, 1993.

[4] Stanley P., "Beginnings and Early Development of Thermoelastic Stress Analysis". Strain, 2008, pp. 285-297.

[5] Žd'árský M., Valach J., "Comparison of strain fields of CT specimen determined by FEM and by thermoplastic measurement". In Engineering Mechanics 2009, pp. 310-319, Prague: UTAM, 2009. ISBN 978-80-86246-35-2. 